\title{
Variable Clinical Phenotypes and Relation of Interferon Signature with Disease Activity in ADA2 Deficiency
}

\author{
Antonella Insalaco, Gian Marco Moneta, Manuela Pardeo, Ivan Caiello, Virginia Messia, \\ Claudia Bracaglia, Chiara Passarelli, and Fabrizio De Benedetti
}

ABSTRACT. Objective. An upregulation of type I interferon (IFN) stimulated genes [IFN score (IS)] was described
in patients with adenosine deaminase 2 deficiency (DADA2). We describe the clinical course of 5
such patients and the role of IS as a marker of disease activity and severity.
Methods. Expression levels of IS were determined by quantitative real-time PCR.
Results. Five white patients were identified as carrying CECR1 mutations. The IS before treatment
was elevated in 4 out of 5 patients and decreased after treatment.
Conclusion. Our data confirm the high variability of DADA2 and suggest type I IS as a biomarker of
disease activity. (First Release January 15 2019; J Rheumatol 2019;46:523-6; doi:10.3899/jrheum.180045)

Key Indexing Terms: INTERFERON

ADA2 DEFICIENCY

DISEASE BIOMARKER

Adenosine deaminase 2 deficiency (DADA2) is an autosomal recessive autoinflammatory disease $\mathrm{e}^{1,2}$ caused by mutations in CECR I (cat eye syndrome chromosome region 1). In some patients, the disease is mild and limited to skin; in others it is severe, with multiorgan involvement including ischemic/ hemorrhagic strokes ${ }^{1,3}$.

An upregulation of type I interferon (IFN) stimulated genes, the so-called type I IFN score (IS), was first described in patients affected by Aicardi-Goutières syndrome (AGS) and in other disorders, including some patients with DADA $2^{5}$.

We report the association of the type I IS with disease activity and severity in 5 patients with genetically confirmed DADA2.

\author{
MATERIALS AND METHODS \\ Characteristics of the patients. The clinical and laboratory features of the \\ patients are described in Table 1. Patients 1 to 4 have been included in the \\ case series by Caorsi, et $a^{3}$. \\ Patient 1. A 12-year-old girl had presented since 3 years of age with recurrent \\ episodes of fever and persistent livedo reticularis of trunk and limbs. An
}

From the Division of Rheumatology, and the Unit of Medical Genetics, Laboratory of Cytogenetics and Molecular Genetics, Institute for Research and Health Care (IRCCS), Ospedale Pediatrico Bambino Gesù, Rome, Italy. A. Insalaco, MD, Division of Rheumatology, IRCCS, Ospedale Pediatrico Bambino Gesù; G.M. Moneta, BSc, Division of Rheumatology, IRCCS, Ospedale Pediatrico Bambino Gesù; M. Pardeo, MD, Division of Rheumatology, IRCCS, Ospedale Pediatrico Bambino Gesù; I. Caiello, BSc, Division of Rheumatology, IRCCS, Ospedale Pediatrico Bambino Gesù; V. Messia, MD, Division of Rheumatology, IRCCS, Ospedale Pediatrico Bambino Gesù; C. Bracaglia, MD, Division of Rheumatology, IRCCS, Ospedale Pediatrico Bambino Gesù; C. Passarelli, PhD, Unit of Medical Genetics, Laboratory of Cytogenetics and Molecular Genetics, IRCCS, Ospedale Pediatrico Bambino Gesù; F. De Benedetti, MD, PhD, Division of Rheumatology, IRCCS, Ospedale Pediatrico Bambino Gesù.

Address correspondence to Dr. A. Insalaco, Division of Rheumatology, Ospedale Pediatrico Bambino Gesù IRCCS, Piazza Sant'Onofrio 4, 00165 Rome, Italy.E-mail: antonella.insalaco@opbg.net

Accepted for publication September 13, 2018. increase in inflammatory markers was observed also during periods of well-being. A skin biopsy showed lymphocytic vasculitis. She was given glucocorticoids (GC), subsequently associated with thalidomide, with recurrence of symptoms following GC tapering. At 9 years, because of persistent recurrent fevers and biochemical inflammation, anakinra and cyclosporine were started but withdrawn because of inefficacy. She developed episodes of arthralgia and chest and abdominal pain, in addition to livedo reticularis.

Patient 2. An 8-year-old girl had presented since she was 6 with recurrent febrile episodes associated with abdominal pain and arthralgia/myalgia, characterized by marked increase of inflammatory measures. Subsequently, livedo developed.

Patient 3. A 13-year-old boy presented at age 1 with recurrent fever, arthralgia, and gastrointestinal symptoms. A colon biopsy revealed nonspecific inflammation. In the subsequent years, he developed arthritis and livedo reticularis of limbs. Increased inflammatory markers were observed also during periods of well-being. At the age of 8 years, GC therapy was started. He presented with seizures, neurosensorial hearing loss, and severe hypertension (HTN). Brain magnetic resonance imaging showed posterior reversible encephalopathy. A kidney computed tomography scan showed a typical triangular area compatible with kidney infarction that explained severe HTN. Further, he developed psoriasis, recurrent episodes of episcleritis, and behavioral disorder.

Patient 4. The younger brother of patient 3, although carrying the same mutations, showed a very mild phenotype characterized by a single episode of prolonged fever with abdominal pain and arthralgia that was resolved with a short cycle of GC therapy.

Patient 5. The patient was evaluated at the age of 18 years. She had presented since 10 years of age with persistent severe livedo reticularis of the limbs. She never presented with other signs or symptoms. Laboratory findings revealed normal inflammatory markers.

Genetic analysis. DNA was extracted from peripheral blood and DNA concentration and purity were quantified by ND-1000 spectrophotometer (NanoDrop; FL $\times 800$ Fluorescence Reader, BioTek). The CECR1 gene (NM_001282225.1) was sequenced through Sanger sequencing (patients 1, 2,5 ) or targeted resequencing (patients 3 and 4) using a customized panel, and analyzed with the NextSeq platform (Illumina). Variants identified by next-generation sequencing have been validated by Sanger. Confirmatory segregation studies were performed on parents.

Gene expression studies. Total RNA was extracted from the blood of patients and of 10 healthy donors (HD) with a PAXgene (PreAnalytix) RNA isolation

Personal non-commercial use only. The Journal of Rheumatology Copyright @ 2019 . All rights reserved. 
Table 1. Genotype, clinical, and laboratory features of the 5 patients with DADA2.

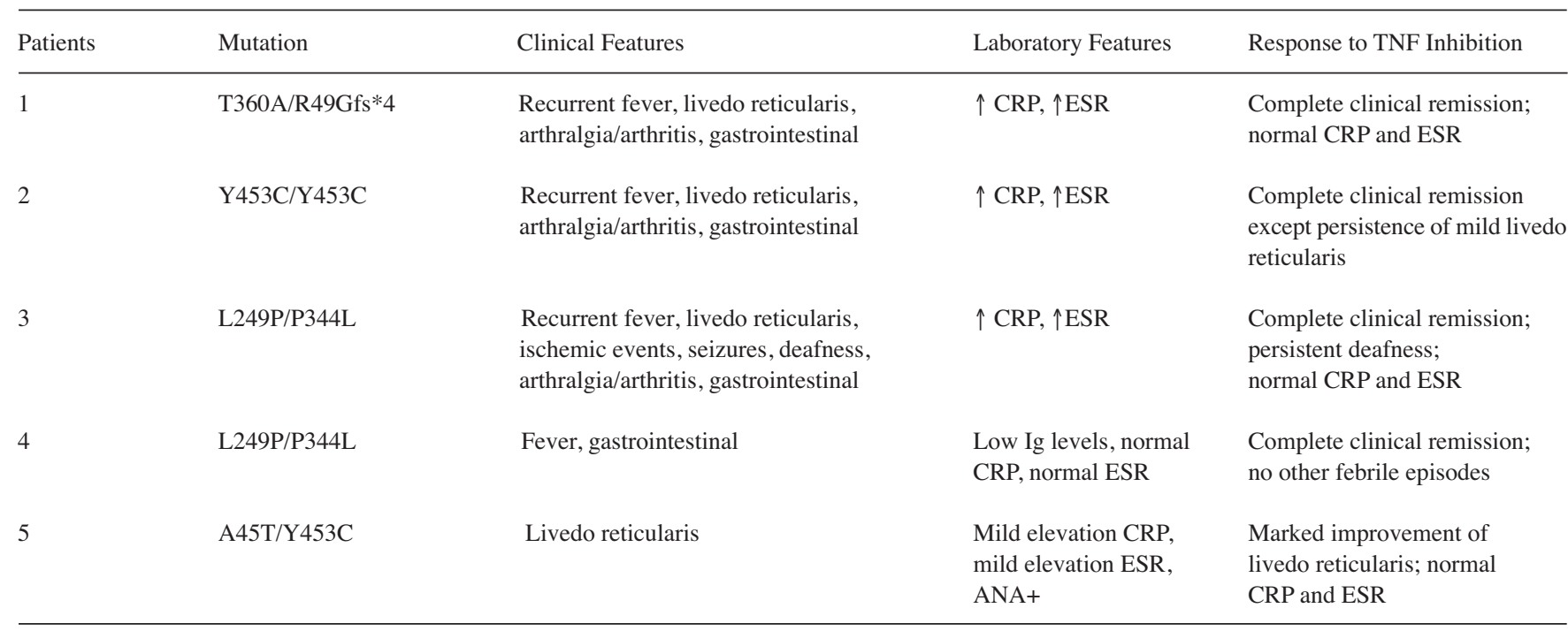

DADA2: adenosine deaminase 2 deficiency; TNF: tumor necrosis factor; CRP: C-reactive protein; ESR: erythrocyte sedimentation rate; Ig: immunoglobulin; ANA: antinuclear antibodies.

kit. Using a Superscript VILO cDNA synthesis Kit (Invitrogen), $2.5 \mu \mathrm{g}$ of total RNA was reverse-transcribed. The type I IFN signature was defined by expression levels of 6 IFN-regulated genes (IFI27, IFI44L, IFIT1, ISG15, RSAD2, and SIGLEC1), measured by quantitative real-time PCR (qRT-PCR), using Taqman universal Master Mix (Applied Biosystems). Expression data were normalized using GAPDH (Applied Biosystems). Expression levels were calculated as $2^{-\Delta \mathrm{Ct}}$. The type I IS was calculated as previously described by Rice and colleagues ${ }^{4,5}$. We considered as positive scores higher than 1.42 (Figure 1).

The study was performed after approval from the ethics committee of the Ospedale Pediatrico Bambino Gesù with the ethics approval number 1486/2017. Signed informed consent was obtained.

\section{RESULTS}

Mutations. Patient 1 carried 2 mutations in the CECR1 gene: a novel frameshift variant, c.144de1G, p.Arg49Glyfs*4, leading to a premature stop, and a missense mutation, c.1078A>G, p. Thr360Ala (rs775440641), already described in DADA2 ${ }^{3}$. Patient 2 is homozygous for c.1358A $>\mathrm{G}$ mutation, p.Tyr453Cys (rs376785840), already described in DADA2 deficiency ${ }^{1,3}$.

Siblings 3 and 4 carried 2 missense mutations: c.746T $>C$ and c.1031C $>\mathrm{T}$, in a compound heterozygous status. These variants have not been reported in other patients. Both p.Leu249Pro (rs757520466), and p.Pro344Leu reside in conserved positions and are predicted to be pathogenic by 2 Insilico software (Insilico Biotechnology) out of 3.

Patient 5 carries 2 missense mutations: the above-mentioned c.1358A $>$ G, p.Tyr453Cys and a c.133C>T, p.Arg45Trp (rs777683953) variant. This has not been previously described, to our knowledge. It is predicted to be pathogenic by 2 Insilico software out of 3 .

Type I IS. Four out of 5 patients had a positive IS (Figure 1B). Patient 4, with normal IS, had only 1 episode of prolonged fever and normal erythrocyte sedimentation rate and
C-reactive protein (CRP). The other 4 patients all had chronic/recurrent symptoms. Patients 1 and 3 (with the highest IS) had more severe and persistent inflammatory features. We correlated the type I IS of each patient with levels of CRP at disease onset and after treatment, finding a significant correlation between the type I IS and CRP $(r=0.73, p<0.05$; data not shown). We have also analyzed the type I IS in other rheumatic disorders (Figure 1B).

All patients were treated with etanercept (ETN), with prompt and complete response. The type I IS decreased markedly in all patients and returned to normal in 2 out of the 4 patients with high IS (Figure 1C). To confirm an activation of type I IFN pathway, we measured plasma levels of CXCL9 and CXCL10, 2 chemokines induced by IFN- $\gamma$ (type II IFN) ${ }^{6}$, in $\mathrm{HD}$ and in DADA2 patients before treatment with ETN. Plasma levels of CXCL9 and CXCL10 were comparable between HD and DADA2 patients, indicating that type II IFN signature is not activated in this cohort of patients (Figure 1D).

\section{DISCUSSION}

DADA2 is a monogenic autoinflammatory disease, characterized by heterogeneous signs and symptoms. Most patients present vasculopathy-related manifestations, such as livedoid skin and ischemic events. Mild autoimmunity and immunodeficiency have been observed in some patients $1,3,7,8,9$. Pure red cell aplasia and myositis were also described at onset ${ }^{10,11}$. A severe clinical picture with prominent cytopenia and lymphoproliferation has also been reported ${ }^{12}$. Recently DADA2 was diagnosed also in patients presenting with immunodeficiency of the B cell compartment ${ }^{13}$. On the other hand, asymptomatic subjects have also been described ${ }^{14}$.

Our patients confirm the phenotypic variability of 

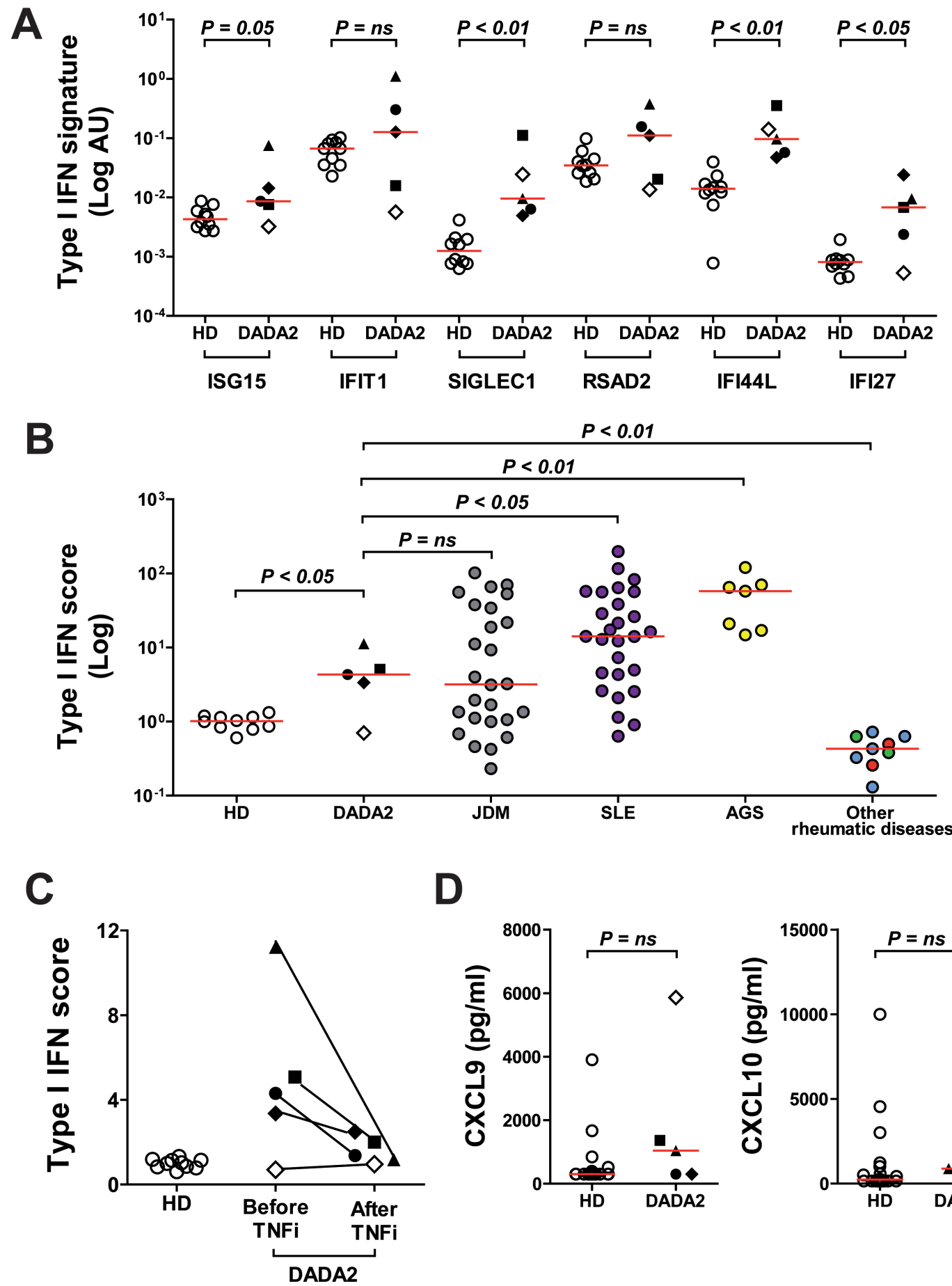

D
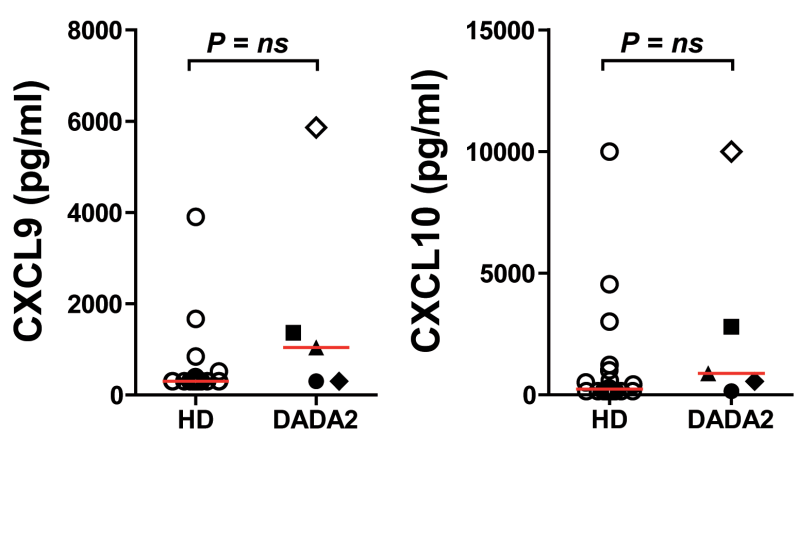

Figure 1. Type I interferon (IFN) signature and type I IFN score (IS) in the peripheral blood of patients with adenosine deaminase 2 deficiency (DADA2). A. Expression levels of the 6 genes typically induced by type I IFN in the peripheral blood of the 5 DADA2 patients $(\mathrm{Pt})$ and HD $(\mathrm{n}=10)$. B. The type I IS in patients affected by DADA2: juvenile dermatomyositis (JDM; $n=26)$, systemic lupus erythematosus (SLE; $n=27)$, Aicardi-Goutières syndrome (AGS; $n=$ 7), and other rheumatic diseases [juvenile idiopathic arthritis (JIA, $n=5$, blue dots), chronic nonbacterial osteomyelitis (CNO, $n=2$, red dots), familial Mediterranean fever (FMF, $\mathrm{n}=2$, green dots)]. In patients affected by SLE and AGS we found high values compared to patients with DADA2. In patients with JDM, values of the type I IS were comparable to those of patients with DADA2. In 9 patients affected by other rheumatic diseases (JIA, CNO, FMF), the type I IS was negative. C. The type I IS in patients with DADA2 before and after treatment with etanercept. D. Plasma levels of CXCL9 and CXCL10 in DADA2 patients compared to HD $(n=21)$. Mann-Whitney $U$ test was used to evaluate statistical differences among groups. We set significance levels at $p<0.05$. Pt 1 $=\mathbf{\Delta} ;$ Pt $2=\mathbf{0}$; Pt $3=\mathbf{\square} ;$ Pt $4=\diamond ;$ Pt $5=\diamond$. Months of treatment for each patient: Pt $1=11$; Pt $2=11$; Pt $3=2.1$; Pt $4=2.1$; Pt $5=1.3$. TNFi: tumor necrosis factor inhibitor.

Personal non-commercial use only. The Journal of Rheumatology Copyright @ 2019 . All rights reserved. 
DADA2. We observed a range of disease severity: from a limited cutaneous vasculitis without systemic inflammation (Patient 5), to severe multisystemic vasculitis in the older of the 2 described brothers (Patient 3 ). The latter presented with multiorgan involvement, including persistent systemic inflammation, deafness, neurological involvement, and malignant HTN, secondary to multiple renal infarctions. Kidney infarctions have never been described in DADA2, to our knowledge. Observation of HTN in a patient with definitive or suspected DADA2 should lead to imaging investigations of the kidneys. Further confirming the wide range of severity, his younger brother, albeit carrying the same genotype, showed a much milder phenotype, with a single episode of prolonged fever, abdominal pain, and arthralgia. However, he is the only patient in this cohort to show a mild hypogammaglobulinemia.

Enhanced IS is present in several diseases such as AGS, systemic lupus erythematosus, and juvenile dermatomyositis $5^{5,15,16}$, in which the activation of the type I IFN pathway has been shown to correlate with disease activity. Recently, an elevated IS was reported in 2 DADA2 patients ${ }^{17}$. In agreement with this observation, we found an increased IS in 4 out of 5 patients. In DADA2, the mechanism leading to the activation of the type I IFN pathway has not been clarified.

The disease shows complete response to tumor necrosis factor (TNF) inhibition ${ }^{3}$. The mechanistic relationship between deficiency of ADA2 and hyperproduction of TNF has not been clarified, and neither has the relationship between TNF and an elevated IS. A cross-regulation between type I IFN and TNF has been hypothesized in some autoimmune disorders ${ }^{18}$.

Whatever the mechanistic relationship might be, it is interesting to note that we found some degree of correlation between disease severity and the degree of elevation of the IS in our patients, suggesting that IS is related to disease severity. Indeed, a normal IS was found only in the patient with a very mild clinical phenotype. In 2 out of 4 patients with positive IS, the score rapidly normalized after treatment (patients 1 and 2), while in the other 2 patients (patients 3 and 5), it decreased.

Our data confirm the high variability of DADA2 regarding age of onset, severity, and organ involvement, even within the same family and among patients with the same mutations. This implies a role for other genetic and epigenetic modifiers, and possibly environmental factors in the disease expres$\operatorname{sion}^{19}$. Further, these data suggest that IS might represent a biomarker of disease activity, severity, and response to treatment in DADA2.

\section{REFERENCES}

1. Zhou Q, Yang D, Ombrello AK, Zavialov AV, Toro C, Zavialov AV, et al. Early-onset stroke and vasculopathy associated with mutations in ADA2. N Engl J Med 2014;370:911-20.

2. Navon EP, Pierce SB, Segel R, Walsh T, Barash J, Padeh S, et al.
Mutant adenosine deaminase 2 in a polyarteritis nodosa vasculopathy. N Engl J Med 2014;370:921-31.

3. Caorsi R, Penco F, Grossi A, Insalaco A, Omenetti A, Alessio M, et al. ADA2 deficiency (DADA2) as an unrecognised cause of early onset polyarteritis nodosa and stroke: a multicentre national study. Ann Rheum Dis 2017;76:1648-56.

4. Rice GI, Forte GM, Szynkiewicz M, Chase DS, Aeby A, Abdel-Hamid MS, et al. Assessment of interferon-related biomarkers in Aicardi-Goutières syndrome associated with mutations in TREX1, RNASEH2A, RNASEH2B, RNASEH2C, SAMHD1, and ADAR: a case-control study. Lancet Neurol 2013;12:1159-69.

5. Rice GI, Melki I, Frèmond ML, Briggs TA, Rodero MP, Kitabayashi $\mathrm{N}$, et al. Assessment of Type I interferon signalling in pediatric inflammatory disease. J Clin Immunol 2017;37:123-32.

6. Groom JR, Luster AD. CXCR3 ligands: redundant, collaborative and antagonistic functions. Immunol Cell Biol 2011;89:207-15.

7. Uettwiller F, Sarrabay G, Rodero MP, Rice GI, Lagrue E, Marot Y, et al. ADA2 deficiency: case report of a new phenotype and novel mutation in two sisters. RMD Open 2016;2: e000236.

8. Kaljas Y, Liu C, Skaldin M, Wu C, Zhou Q, Lu Y, et al. Human adenosindeaminases ADA1 and ADA2 bind to different subsets of immune cells. Cell Mol Life Sci 2017;74:555-70.

9. Schepp J, Bulashevska A, Mannhardt-Laakmann W, Cao H, Yang F, Seidl M, et al. Deficiency of adenosine deaminase 2 causes antibody deficiency. J Clin Immunol 2016;36:179-86.

10. Zavialov AV, Yu X, Spillmann D, Lauvau G, Zavialov AV. Structural basis for the growth factor activity of human adenosine deaminase ADA2. J Biol Chem 2010;285:12367-77.

11. Hashem H, Egler R, Dalal J. Refractory pure red cell aplasia manifesting as deficiency of adenosine deaminase 2. J Pediatr Hematol Oncol 2017;39:293-6.

12. Van Montfrans JM, Hartman EA, Braun KP, Hennekam EA, Hak EA, Nederkoorn PJ, et al. Phenotypic variability in patients with ADA2 deficiency due to identical homozygous R169Q mutations. Rheumatology 2016;55:902-10.

13. Schepp J, Proietti M, Frede N, Buchta M, Hübscher K, Rojas Restrepo J, et al. Screening of 181 patients with antibody deficiency for deficiency of adenosine deaminase 2 sheds new light on the disease in adulthood. Arthritis Rheumatol 2017;69:1689-700.

14. Nanthapisal S, Murphy C, Omoyinmi E, Hong Y, Standing A, Berg $\mathrm{S}$, et al. Deficiency of adenosine deaminase type 2: a description of phenotype and genotype in fifteen cases. Arthritis Rheumatol 2016;68:2314-22.

15. Baechler EC, Batliwalla FM, Karypis G, Gaffney PM, Ortmann WA, Espe KJ, et al. Interferon-inducible gene expression signature in peripheral blood cells of patients with severe lupus. Proc Natl Acad Sci U S A 2003;100:2610-5.

16. Niewold TB, Kariuki SN, Morgan GA, Shrestha S, Pachman LM. Elevated serum interferon-alpha activity in juvenile dermatomyositis: associations with disease activity at diagnosis and after thirty-six months of therapy. Arthritis Rheum 2009; 60:1815-24.

17. Skral-Baumgartner A, Plecko B, Schimdt WM, Konig N, Hershfield $\mathrm{M}$, Gruber-Sedlmayr U, et al. Autoimmune phenotype with type I interferon signature in two brothers with ADA2 deficiency carrying a novel CECR1 mutation. Pediatr Rheumathol Online J 2017;15:67.

18. Cantaert T, Baeten D, Tak PP, van Baarsen LG. Type I IFN and $\mathrm{TNF} \alpha$ cross-regulation in immune-mediated inflammatory disease: basic concepts and clinical relevance. Arthritis Res Ther 2010;12:219.

19. Meyts I, Aksentijevich I. Deficiency of adenosine deaminase 2 (DADA2): updates on the phenotype, genetics, pathogenesis, and treatment. J Clin Immunol 2018;38:569-78. 